\section{Underreported use of non-steroidal anti-inflamma- tory drugs in an outpatient gastroenterology practice: a prospective office-based survey}

\author{
Raj Majithia, ${ }^{1}$ David A. Johnson, ${ }^{2}$ \\ Michael J. Ryan, ${ }^{2}$ F. Taylor Wootton, ${ }^{2}$ \\ Jeff Willis, ${ }^{2}$ Kelvin Hornbuckle, ${ }^{2}$ \\ Whitney Brooks ${ }^{2}$ \\ ${ }^{1}$ Washington Hospital Center, Division \\ of Gastroenterology, Washington DC; \\ 2Division of Gastroenterology, Eastern \\ Virginia Medical School, Norfolk, VA, \\ USA
}

\section{Abstract}

Non-steroidal anti-inflammatory drugs (NSAIDs) are one of the most commonly used class of medications worldwide. It is estimated that more than 20 million people take NSAIDs daily. We examined the underreported use of NSAIDs on a standardized office intake assessment questionnaire of patient reported medications. A questionnaire was administered to patients following written and verbal confirmed report of current medications to nursing staff. One hundred consecutive patient intake assessments were evaluated from the patient intake sheets for a busy private gastroenterology specialty practice. After patients listed their current medications, they were given a survey and asked to acknowledge any use of 30 non-prescription formulations that contained NSAIDs. Patients filled these surveys while waiting for the health care provider who then reviewed the response accuracy with the patient during the visit. Changes in the patient's initial and subsequently corroborated use or non-use of NSAIDs were recorded and tabulated. The educational level of all participants was also assessed. Of the 100 surveys completed, $6 \%$ of patients had not completed high school, 19\% had completed high school, $8 \%$ had started but not finished college, $54 \%$ had completed college, $2 \%$ had started but not finished graduate school, and 11\% had completed graduate school. The educational level of the patients did not correlate with underreporting of NSAID usage. On the survey, 18\% of patients noted use of an NSAID listed that had not been reported verbally to nursing staff; $8 \%$ of those patients reported daily use, $15 \%$ weekly use and $76 \%$ reported use within the last month. Patients were then asked in the questionnaire as to the reason for not reporting medication intake to nurse; $14 \%$ stated they were never asked about the specific medication, $22 \%$ did not think the medications were important enough to list, $34 \%$ bought the medication with prescriptions, and $30 \%$ noted their reason as their doctor did not prescribe the medication to them. Using a prospective questionnaire in a private practice gastrointestinal office, these data show that patients underreport use of non-prescription NSAIDs. Based on the data, it is clear that this underreporting can have a detrimental effect on procedure related as well as long-term patient care. Future studies would be needed to examine the incidence of side effects due to underreporting of NSAIDs.

\section{Introduction}

The Center for Disease Control approximates that nearly 20 million Americans use various non-steroidal anti-inflammatory drugs (NSAIDs) on a daily basis. These drugs are used for a wide variety of conditions and many are available as over-the-counter drugs without prescription. One study estimates that from 5 to 7 percent of hospital admissions are related to adverse effects of drugs, and of these hospitalizations, those that result from gastrointestinal, nervous system, renal, or allergic effects of aspirin or non-aspirin NSAIDs are responsible for approximately 30 percent. $^{1}$

While NSAID use has been proven beneficial in alleviating symptoms from many inflammatory conditions, their use is not without adverse effects. Gastrointestinal toxicity including dyspepsia, peptic ulcer disease and bleeding are commonly seen. In a study of the association of NSAIDs and gastrointestinal bleeding, the relative risk of symptomatic ulcers for NSAID users were 4.0 as compared to 1.03 for non-users. ${ }^{2}$ In addition to gastrointestinal toxicity, NSAIDs have also been noted to cause hepatic injury, cardiovascular, pulmonary, hematologic, and central nervous system effects. ${ }^{3}$ This not only directly affects the patient, but has a significant cost burden on health care. The incidence rate of NSAID induced gastropathy has been estimated at $1 \%$ in patients taking NSAIDs for three to sixmonths and approximately $4 \%$ in patients on NSAID therapy for greater than a year. The annual health care costs for NSAID-induced gastropathy are estimated at 4 billion Dollars. ${ }^{4}$

The efficacy, use and adverse effects of NSAIDs have been well documented; however, as many of these medications are over the counter, the underreported use of NSAIDs has not been established. In clinical practice, it is important that physicians are aware of all medications their patients take. In this quality improvement project, we examined the underreported use of NSAIDs using a preliminary
Correspondence: David A. Johnson, Division of Gastroenterology, Eastern Virginia Medical School, 885 Kempsville Road, Suite 114, Amelia Building, Norfolk, VA 23502, USA.

Tel. +1.757.4660165 - Fax: +1.757.4669082

E-mail: dajevms@aol.com

Key words: NSAIDs, outcomes research, gastroenterology practice, medication reconciliation.

Conflict of interests: the authors declare no potential conflict of interests.

Received for publication: 14 August 2011.

Revision received: 8 October 2012.

Accepted for publication: 3 December 2012.

This work is licensed under a Creative Commons Attribution NonCommercial 3.0 License (CC BYNC 3.0).

(C) Copyright R. Majithia et al., 2013

Licensee PAGEPress, Italy

Gastroenterology Insights 2013; 5:e3

doi:10.4081/gi.2013.e3

office intake assessment questionnaire of patient-reported medications.

\section{Materials and Methods}

This study was undertaken by the practice as a quality improvement project to assess practice standards concerning medication intake from patients. The patients were given an intake form upon arrival and registration to the practice. On the form, the patients were asked to fill out any and all medications they were taking. The form specified to document both prescription and over the counter medications. As per practice protocol, patient medications were verbally reviewed by the office nurse while the patient is in the visit room. The nurse would confirm listed medications with the patient in a verbal review while also documenting the medications on an intake form. The patient was also questioned as to the level of education achieved, defining the highest degree at the time of final graduation or the highest level started but not completed. Subsequently while waiting for the health care provider (physician or nurse practitioner), 100 consecutive patients were asked to fill out a subsequent questionnaire focusing on the ingestion of 30 OTC medications which are NSAIDs, or medications that have a component ingredient thereof (Figure 1). Subsequently during the health care provider visit, these questionnaires were compared by the health care provider to verbally-obtained medication lists and reviewed with the patient and validated for accuracy. 


\section{Sample size/statistical analysis}

As this was a descriptive study or patient reporting, there was no specific sample size calculation. Rather the data on 100 consecutive patients was construed as a sample for a descriptive analysis. Covariable effects for not reporting NSAID use was assessed by univariable and multivariable regression analysis with analysis of age, gender, frequency of use, level of education, and the patient's reason for not reporting usage on initial questioning.

\section{Results}

On the self-reported medication survey, $18 \%$ of patients noted use of an NSAID listed that had not been reported verbally to nursing staff $(n=100)$. Eight percent of those patients reported daily use, $15 \%$ weekly use, and $76 \%$ reported use within the last month (Figure 2).

In addition to these results, patients were asked their reason for not originally reporting NSAID use to the intake nurse. Fourteen percent stated they were never asked about the specific medication, $22 \%$ did not think the medications were important enough to list, $34 \%$ bought the medication with prescriptions, and $30 \%$ noted their reason as their doctor did not prescribe the medication to them (Figure 3 ). Neither age nor gender was a significant factor associated with this underreporting.

Of the 100 respondents, $6 \%$ of patients had not completed high school, 19\% had completed high school, $8 \%$ had started but not finished college, $54 \%$ had completed college, $2 \%$ had started but not finished graduate school, and $11 \%$ had completed graduate school. The educational level of the patients did not correlate with underreporting of NSAID usage as our patient population was a relatively well-educated homogenous group.

\section{Discussion and Conclusions}

In this quality improvement project, we examined the underreported use of NSAIDs using a preliminary office intake assessment questionnaire of patient-reported medications. This project is the first that demonstrates the use of NSAIDs by patients without reporting this use to physicians. We found that nearly 1 out of 5 patients did not report the use of NSAIDs with $8 \%$ of these patients using NSAIDs daily. Of these patients, it is important to recognize that nearly $22 \%$ of patients noted that they did not feel the medications were important enough to report to their physician and an addi- tional 30\% did not report the NSAIDs because their doctor did not prescribe them. While NSAIDs are proven to be effective in treating certain conditions, it is imperative that patients and physicians realize the importance of reporting all medication use including herbal, vitamin, prescribed and over-the-counter. It is clear that patients do not understand the adverse effects of NSAIDs and that some of these can be predicted or prevented by physicians if they report all medication use to their doctor.

These findings of unreported usage are particularly relevant considering the reported scope of usage of NSAIDs. The Food and Drug Administration reports that greater than 20 million people use NSAIDs daily. NSAIDs accounted for 70 million prescriptions over one year, with an additional 30 billion over the counter doses sold annually. ${ }^{5}$ With the ever aging population, it is thought that this number is expected to increase. It is estimated that nearly 7 out of 10 adults over the age of 65 are using NSAIDs weekly. These drugs are both easily available and can be very effective, which leads to a misperception about the safety of these medications. Without reporting NSAID use to physicians, patients are exposing themselves to adverse effects. Physicians cannot properly prepare or be aware that such effects exist without knowing the medications their patients are taking. A British study examined emergency room

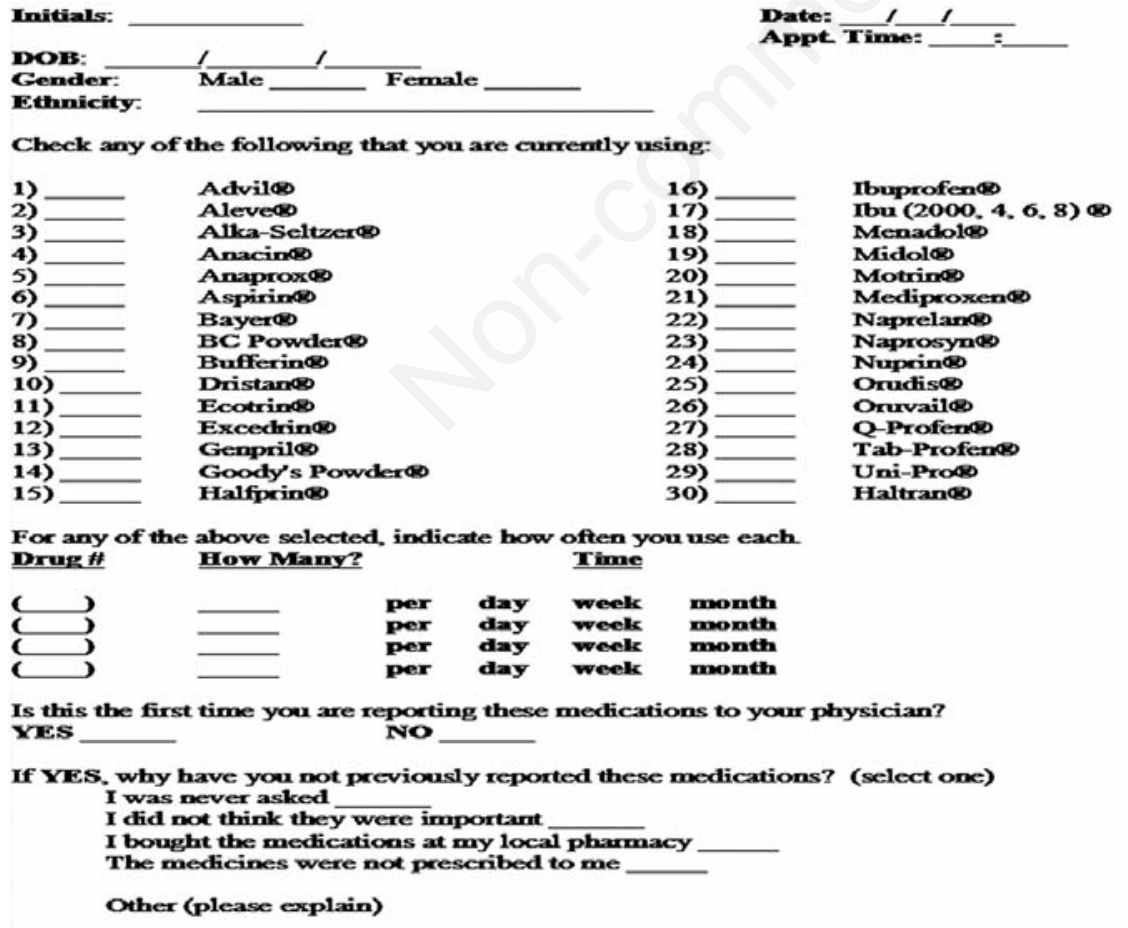

Figure 1. Questionnaire given to patients assessing the ingestion of 30 OTC medications which are non-steroidal anti-inflammatory drugs, or medications that have a component ingredient thereof.

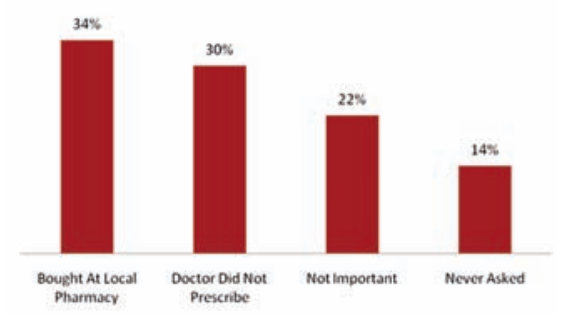

Figure 2. This chart identifies the frequency of non-steroidal anti-inflammatory drugs use among our patient population based on questionnaire results.

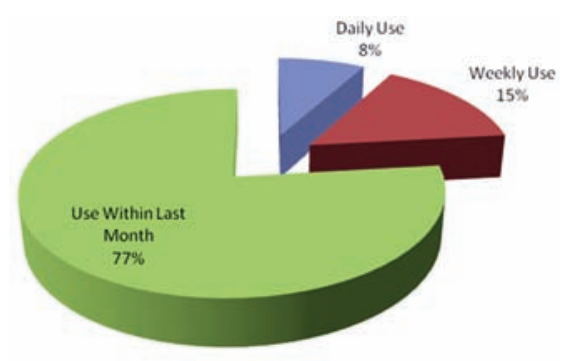

Figure 3. This chart depicts the reasons given by patients for the under-reporting of Non-steroidal anti-inflammatory drugs use. 
admissions for upper GI bleeds with respect to NSAID use. ${ }^{6}$ This study was a retrospective case-control survey using 620 emergency admissions over one year, with controls for 460 cases. Controls were matched for sex, age, and date of admission. Overall results showed that blood transfusion was required in $36 \%$ of all cases, and in $50 \%$ of those taking NSAIDs. NSAID users needed significantly more blood transfused than non-users. NSAID users also required a significantly longer stay ( $24 \%$ had a hospital stay of more than 14 days). NSAID users overall mortality was $20 \%$ compared to $14 \%$ in non-users.

One study showed that $7 \%$ of men and $12 \%$ of women used prescription NSAIDs, while $20 \%$ of men and $30 \%$ of women used non-prescription NSAIDs. ${ }^{7}$ The increased availability and use of NSAIDs is not without risk. The widespread use of NSAIDs has meant that adverse effects have become more prevalent to practitioners and have been documented as contributors to adverse drug reactions including gastrointestinal, renal, cardiovascular, pulmonary, hematologic, central nervous system, dermatologic and even effects on pregnancy.

The main adverse effect of NSAID use is direct and indirect irritation of the gastrointestinal tract. Aspirin and many other NSAIDs are carboxylic acid derivatives that are not ionized by the acidity of gastric $\mathrm{pH}$ and can therefore be absorbed easily across the gastric mucosa. Once absorbed, the drug becomes ionized and trapped which can cause irreversible damage to gastric epithelial cells. ${ }^{8}$ This is considered the direct irritation. In addition, the gastric epithelial injury caused by NSAIDs is also seen with intravenous use suggesting that an indirect source of damage is also present. ${ }^{9}$ Inhibition of COX-1 by NSAIDs decreases production of prostaglandins reducing their protection of the gastrointestinal tract. Common effects include nausea, vomiting, dyspepsia, gastric ulceration and bleeding, and diarrhea. ${ }^{10}$ NSAID-associated dyspepsia occurs in up to $50 \%$ of patients who use these drugs. The risk of ulceration with medication use increases with duration of therapy and with higher doses. Because gastric mucosa uses COX-1 to generate mucosal-protective prostaglandins, Aspirin doses as low as 10 $\mathrm{mg} /$ day can inhibit the generation of the protective barrier. ${ }^{11}$ Only $1 \%$ to $3 \%$ of patients develop serious gastrointestinal side effects while taking NSAIDs. Despite this low percentage, it is important to remember the large pool of patients taking NSAIDs on a regular basis. With those numbers, the percentage results in substantial NSAID related morbidity and mortality each year. It is estimated that NSAID-induced gastrointestinal complications result in approximately 16,500 deaths and more than 100,000 hospitalizations, with costs exceeding $\$ 2$ billion annually. In a report of cost of adverse effects of NSAIDs, researchers found that the average annual costs were $\$ 1234$ in NSAID users compared with $\$ 1036$ for controls. ${ }^{12}$ After adjusting for socio-demographic factors, other health care utilization, and relevant comorbid diseases, the average annual cost for the major NSAID related adverse effects studied was $\$ 117$ higher for patients filling a NSAID prescription. Given this unexpectedly high prevalence of non-reported use of NSAIDs, there is a significant unrecognized potential for NSAID related complications in particular with patients having other recognized risks such as steroids, anticoagulants, antiplatelet agents or other NSAIDs. ${ }^{13,14}$ It was particularly notable in this evaluation that when $30 \%$ of patients were asked their reason for not originally reporting NSAID use to the intake nurse they informed that their gastroenterology doctor did not prescribe the medication to them. It is evident that the responsibility for medication intake should be more clearly shared between physicians as well as well-informed patients. Fourteen percent of patients stating their reason for under-reporting OTC NSAID usage was the lack of specific questions on the intake information requested. We would suggest there is a potential significant need for more accurate mechanisms for specific assessment to more clearly define the patient risk profile. From the results of this quality improvement project, it appears that patients may under-report use of NSAIDs in an outpatient gastrointestinal practice. Given the potential NSAID related risks, it is important to recognize the potential implications of under-reporting this medication usage. As an accurate reporting of NSAID usage would be important to record for most patients who are followed in a gastroenterology practice, it seems that there may be a need for more comprehensive methods of tracking and recording of the usage of all medications - in particular the OTC formulations. Some practices encourage patients to carry a list of medications that can be updated by the physician for each visit. This would be ideal if patients listed each pill taken and not just what the patient perceives as a medication are recorded. This may help both the doctor and patient understand each medicine and better define the adverse effect potential and make appropriate interventions or counseling to the patient in attempt to minimize the potential for adverse outcomes. Ultimately, accuracy in the reporting communication is the key. Specific listing of OTC medications containing NSAIDs may however, be helpful for the health care provider's more accurate determination of relative risks related to the otherwise unrecognized or under-reported use of these medications. Future studies would be needed to examine the health care provider alteration of health care plans (e.g. counseling, medication alteration /addition/discontinuance) as well as incidence of side effects or complications directly attributable to underreporting of NSAIDs

\section{References}

1. Pirmohamed M, James S, Meakin S, et al. Adverse drug reactions as cause of admission to hospital: prospective analysis of 18,820 patients. BMJ 2004;329:15.

2. Garcia Rodriguez LA, Hernandez-Diaz S. Risk of uncomplicated peptic ulcer among users of aspirin and nonaspirin nonsteroidal antiinflammatory drugs. Am J Epidemiol 2004;159:23-31.

3. Knodel LC. NSAID adverse effects and interactions. Who is at risk? American Pharmacy 1992;NS32:39-47.

4. Adams EH. Preventing Complications of non-steroidal antiinflammatory drug therapy. Available from: http://rxweb.ulm.edu/ pharmacy/oore/hc\%20provider\%20p/NSAID \%20P2.pdf. Accessed: January 2013.

5. Green GA. Understanding NSAIDs: from aspirin to COX-2. Clinical Cornerstones 2001;3:50-60.

6. Blower AL, Brooks A, Fenn CG, et al. Emergency admissions for upper gastrointestinal disease and their relation to NSAID use. Aliment Pharmacol Ther 1997;11:28391.

7. Tamblyn R, Berkson L, Dauphinee WD, et al. Unnecessary prescribing of NSAIDs and the management of NSAID-related gastropathy in medical practice. Ann Intern Med 1997;127:429-38.

8. Flower R. The development of COX2 inhibitors. Nat Rev Drug Discov 2003;2:179.

9. Estes LL, Fuhs DW, Heaton AH, et al. Gastric ulcer perforation associated with the use of injectable ketorolac. Ann Pharmacother 1993;27:42.

10. Carson JL, Strom BL, Duff A, et al. Safety of nonsteroidal antiinflammatory drugs with respect to acute liver disease. Arch Intern Med 1993;153:1331.

11. Wallace JL. Prostaglandins, NSAIDs, and gastric mucosal protection: why doesn't the stomach digest itself? Physiol Rev 2008;88: 1547-65.

12. Solomon DH, Glynn RJ, Bohn R, et al. The hidden cost of nonselective nonsteroidal antiinflammatory drugs in older patients. $\mathrm{J}$ Rheumatol 2003;30:792-8.

13. Piper JM, Ray WA, Daugherty JR. Corticosteroid use and peptic ulcer disease: role of nonsteroidal anti-inflammatory drugs. Ann Intern Med 1991;114:735.

14. Taha AS, Angerson WJ, Knill-Jones RP, et al. Upper gastrointestinal haemorrhage associated with low-dose aspirin and antithrombotic drugs - a 6-year analysis and comparison with non-steroidal anti-inflammatory drugs. Aliment Pharmacol Ther 2005;22:2859 . 